\title{
Soft lonization Mass Spectrometry for Studying Noncovalent Interactions
}

\author{
Renato Zenobi*
}

\begin{abstract}
Biochemistry and structural biology have benefited tremendously from the development of electrospray ionization and matrix-assisted laser desorption/ionization, the two most important soft ionization methods used in modern mass spectrometry. Of importance in this context is that noncovalently bound complexes can be maintained in the gas phase using appropriate sample preparation conditions and instrumental settings in ESIMS and MALDI-MS. This allows one to determine the architecture and stoichiometry of large noncovalent complexes, the binding of small molecule ligands and cofactors, and often even noncovalent binding affinities, since bound and unbound states can be clearly resolved and quantified using MS. This article presents an overview of the research in this particular area undertaken by my research group at ETH Zurich. I will introduce a number of specialized technologies that we have available in our laboratory, including MALDI mass spectrometry with high-mass detection for measuring complexes with molecular weights in the 100s of kDa range; the so-called 'gap sampler' technology for high-throughput experiments; and a temperature programmed spray source for ESI. A number of selected applications will then be presented, including the study of GPCRs and other membrane proteins and their complexes, the determination of the melting behavior of non-canonical nucleic acid structures, the investigation of heterogeneous protein complexes and protein-based capsids, and the investigation of the influence of protein post-translational modifications on noncovalent binding profiles.
\end{abstract}

Keywords: ESI-MS · MALDI-MS · Noncovalent complexes · Soft ionization mass spectrometry

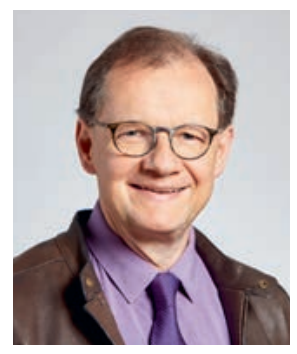

Renato Zenobi is professor for Analytical Chemistry at ETH Zurich. $\mathrm{He}$ obtained his education at ETH Zurich and Stanford University, worked at Pittsburgh University, the University of Michigan (Ann Arbor), and EPFL before taking on a faculty position at ETHZ in 1995. $\mathrm{He}$ is best known for using modern mass spectrometry methods to solve problems in the life sciences, for his contributions to understanding ion formation mechanisms in electrospray and laser mass spectrometry, and for the invention of tipenhanced Raman spectroscopy, a method that allows one to gain detailed molecular information on the nanometer scale. Together with clinicians, he has developed a mass spectrometric 'chemical nose' that could revolutionize medical diagnosis. He is the recipient of numerous fellowships and awards, most recently the 2015 Fresenius Award (German Chemical Society), a 2017 ERC Advanced Grant from the EU, and the 2019 'Golden Owl' from ETHZ for excellence in teaching. Zenobi also enjoys sports (skiing, ski mountaineering, running, biking, unicycling), and is a semi-professional violinist, performing regularly in concerts. He plays orchestral and chamber music as well as gipsy jazz.

\section{Introduction}

Electrospray ionization (ESI) and matrix-assisted laser desorption/ionization (MALDI), the two preeminent soft ionization mass spectrometry (MS) methods, have become key for conducting biochemical and structural research. Using near-native solution conditions and soft instrument settings, these techniques al-

${ }^{*}$ Correspondence: Prof. Dr. R. Zenobi, E-mail: zenobi@org.chem.ethz.ch Department of Chemistry and Applied Biosciences, ETH Zurich,

Vladimir-Prelog-Weg 3, CH-8093 Zurich, Switzerland low for the observation of intact noncovalently bound complexes in the gas phase. Data obtained from such studies can be used to elucidate structural questions of biomolecular complexes, e.g., subunit stoichiometries and ligand-induced allosteric effects, and in many cases allow biomolecular binding affinities to be measured. The past years have witnessed many exciting, fundamental scientific developments in this area, but soft ionization MS is becoming equally important for applied science, like quality assurance of antibodies and biosimilars via detailed characterization of their glycosylation patterns, or studying potential drug molecules, often small ligands bound to higher-order complexes. The field has been reviewed several times during the past years, and key information can be found in some recent review articles. ${ }^{[1-10]}$ This contribution will focus on research done in the Zenobi laboratory at ETH Zurich. First a few technologies will be introduced that are not commonplace and allow our research group to conduct studies that cannot be done with commercial equipment. This will then be followed by the presentation of several applications of soft ionization mass spectrometry to the investigation of noncovalent complexes, using both MALDI-MS and native ESI-MS.

\section{Special Technologies}

Our work relies on several unique technologies that are available in our laboratory, including but not limited to: chemical crosslinking followed by MALDI-MS with high-mass detection, a technology we use to study large multi-subunit protein complexes; the so-called 'gap sampler', a device that allows very rapid injection of nanoliter sample volumes into an ESI-MS instrument; ${ }^{[11-13]}$ and temperature-controlled nanoESI as well as temperature-jump ESI sources, which allow for the measurement of thermodynamic and kinetic parameters of noncovalent interactions in unprecedented detail, especially on equilibria involving several components that cannot be distinguished by standard biophysical techniques. ${ }^{[14]}$ These capabilities also make us soughtafter collaboration partners. 


\subsection{High-mass MALDI Mass Spectrometry}

MALDI predominantly produces ions with low charge states, hence the $m / z$ of high-mass molecules can be very high, easily in the range of 100s of $\mathrm{kDa}$. The regular detectors used in MALDI time-of-flight mass spectrometers, microchannel plates, are unsuitable to detect such high molecular weight, slow flying ions. An alternative is to use ion-to-ion conversion, on a dynode detector for sensitive detection of ions with $m / z$ above $100 \mathrm{kDa}$. Such ion conversion detectors (ICDs) include a collision dynode and a secondary electron multiplier. When an ion impacts the surface of the ICD, secondary ions are generated. These secondary ions are typically much smaller ( 100 Da) than the original high-mass ions and thus much easier to be accelerated in a secondary high voltage $(20 \mathrm{kV})$ acceleration field. Finally, the secondary ions are detected by a secondary electron multiplier.

Another problem of MALDI is that it is less soft than ESI, and especially high-mass noncovalent complexes may dissociate during the MALDI process. Such complexes can be chemically stabilized using crosslinking strategies. We recently developed an absolute quantitative MALDI-MS strategy that combines chemical crosslinking with calibration by an internal standard to quantify protein protein interactions. ${ }^{[15]}$ As standards, commercially available, purified proteins ( $\beta$-galactosidase, $\beta$-Gal, and bovine serum albumin, BSA) were employed. The homogeneity and stability of different methods for depositing the sample and the internal standard were carefully evaluated, eventually resulting in a sandwich method with a saturated sinapinic acid as the top layer performing the best for quantification of proteins and their complexes using MALDI-MS. Chemical crosslinking was used to stabilize the PPIs in their equilibrium state.

\subsection{The Gap Sampler}

Developed by C. Fattinger at Hoffmann-LaRoche, ${ }^{[16]}$ our group has been operating an autosampler for ESI called the Capillary Gap Sampler, a miniaturized sampling device that can be used as a platform for direct and fast uptake or extraction ${ }^{[17]}$ of $\mathrm{nL}$ volumes of sample. Briefly, it uses a solid stainless-steel pin for sampling a tiny droplet of liquid from a sample solution by brief immersion, followed by release of the liquid in a flowing stream of ESI buffer, either in a liquid meniscus formed in the gap between two capillaries, or in a cavity micromachined into a capillary. The sampling pin is cleaned automatically before being immersed into the next sample. With the flowing stream, the sample is then transported to an ESI spray needle, ionized, and analyzed in a mass spectrometer. The system is always ready for receiving new samples and allows fast (9-11 s) and automated analyses in a high-throughput fashion.

\subsection{Temperature-programmed and Temperature Jump ESI Mass Spectrometry}

Our group has also made significant advances based on the use of the temperature-controlled (TC) and temperature jump ESI sources. The most common TC-ESI-MS sources use heated metal blocks to control temperature, with several designs being used by different researchers (e.g., in the Robinson lab ${ }^{[18]}$ or the Kaltashov $\left.l a b^{[19]}\right)$. Recently, a source that uses a three-tier Peltier design was described in the literature by McCage et al., ${ }^{[20]}$ which allows for the spray solutions to be set between 5 to $98^{\circ} \mathrm{C}$, with an accuracy of $\pm 2{ }^{\circ} \mathrm{C}$. Such nESI sources were used to investigate effects such as conformational changes occurring during protein thermal denaturation (e.g., cytochrome $\left.\mathrm{c}^{[21]}\right)$, and to elucidate intermediate stable structures of ubiquitin ${ }^{[22]}$ and bovine serum albumin. ${ }^{[23]}$ The intermediate states of DNA complexes have also been investigated using a similar design coupled to ion mobility spectroscopy. [24]

Our laboratory employs two different types of temperaturecontrolled ESI spray sources, one that is similar to other TC-ESIMS sources, and another one that allows temperature jumps to be applied to a flowing sample (see Fig. 1). The latter design has been shown to be a useful tool for probing both the thermodynamics and kinetics of ligand binding, of multimer formation, and of melting of non-canonical nucleic acid structures, as shown in Marchand et al. ${ }^{[14]}$

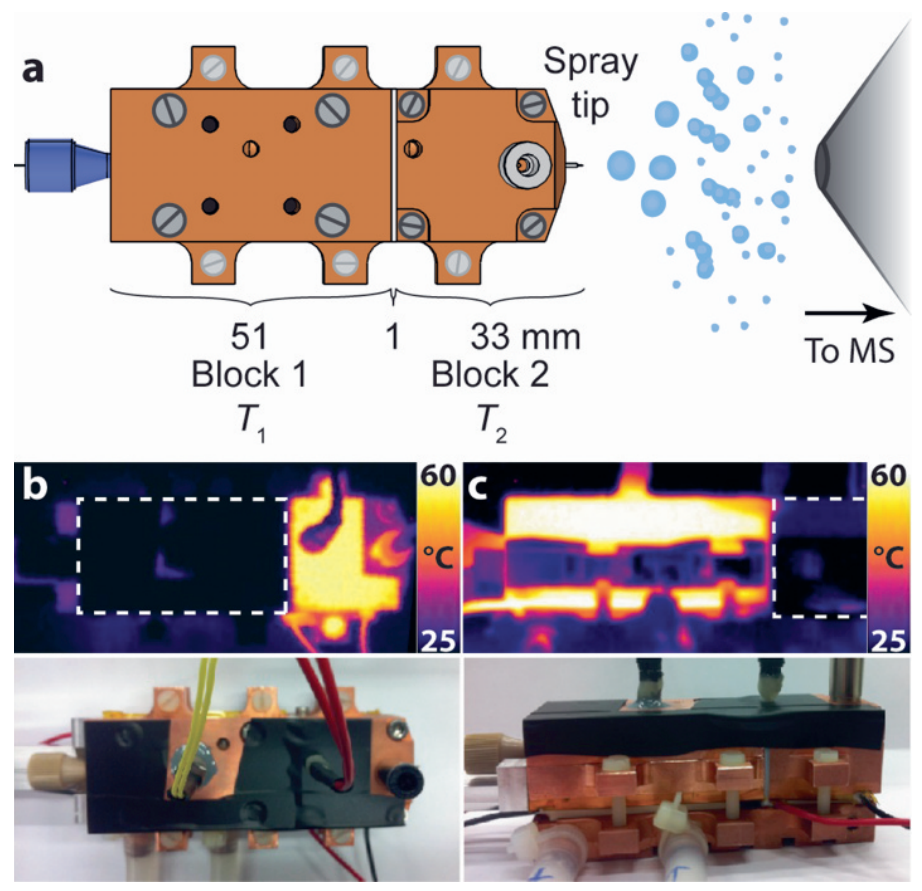

Fig. 1. (a-c) A temperature-jump ESI source. The design shown in (a) subjects a flow of sample to a temperature jump, either from cold to hot (b), for example, for following 'melting' of a complex, or from hot to cold (c). The flow rate determines how much time the sample spends in the hot and cold regions of the device, and measurements with different flow rates allow one to get kinetic information. Reprinted with permission from Nature Commun.

\section{Studies of Membrane-bound Proteins and Protein Complexes by MALDI-MS}

The study of membrane-bound proteins and membrane protein complexes, including GPCRs and their partner proteins is one of our group's most important contributions to the study of noncovalent complexes by MALDI-MS. We are able to determine the molecular weight of membrane proteins and membrane protein complexes and to obtain stoichiometric information, for example of the binding between membrane protein and nanobodies. For example, as shown in Fig. 2, useful information could be obtained about the stoichiometry of PglK, a homodimeric $\mathrm{ABC}$ transporter interacting with a stabilizing nanobody used for locking the membrane protein in a certain conformation before analysis by X-ray crystallography ${ }^{[25]}$ (Fig. 2). To prevent dissociation during the MALDI process, we use stabilization via chemical cross-linking to keep the complexes intact.

Details about the molecular interplay between GPCRs and their G-protein partners, the first step of GPCR downstream signal transduction, remains difficult to unravel. Using chemical crosslinking and high-mass MALDI-MS, the GPCR $\bullet$ G-protein complex was detected, allowing us to investigate the effect of ligands on GPCR conformation and on coupling selectivity. Over 70 ligand $\bullet$ GPCR•partner protein combinations were investigated in this study, which employed three GPCRs (rhodopsin, beta-1 adrenergic receptor [ $\beta 1 \mathrm{AR}]$, and angiotensin II type 1 receptor) as well as engineered $\mathrm{G} \alpha$ proteins ( $\mathrm{mGs}, \mathrm{mGo}, \mathrm{mGi}, \mathrm{mGq}$ ) and nanobody 80.[26] Furthermore, we developed a normalization strategy[27] for the quantitative measurement of GPCRs binding 

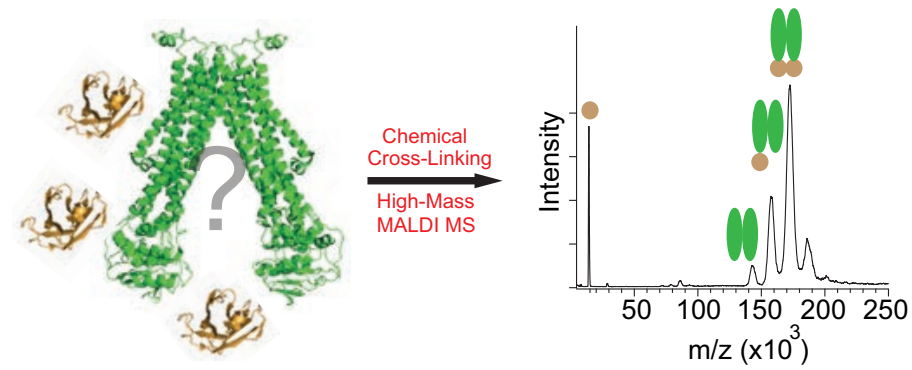

Fig. 2. Stoichiometry of a membrane protein (PglK, green; PDB: 5C76) in complex with stabilizing nanobodies (brown; for illustration PDB: $113 \mathrm{~V}$ was used), detected by high-mass MALDI-MS. Reproduced with permission from ref. [25], M. Köhler et al., Anal. Chem. 2018, 90, 5306. Copyright 2018, American Chemical Society.

affinities with partner proteins, using only picomoles of protein. This quantification method was applied to $\beta 1 \mathrm{AR}$, which uncovered an allosteric effect of $\mathrm{Nb} 80$ binding, which assisted in displacing nadolol for isoprenaline. We also quantified complex formation with wild-type heterotrimeric $G \alpha_{i} \beta \gamma$ and $\beta$-arrestin 1, showing carvedilol induces increased coupling of $\beta$-arrestin 1 and Goi $\beta \gamma$ to $\beta 1 \mathrm{AR}$. An example is shown in Fig. 3. the characterization of the isoprenoid biosynthesis enzyme IspF from Arabidopsis thaliana (AtIspF), a homotrimeric protein complex with multiple binding sites for several types of ligands, including a metal cofactor and a synthetic inhibitor. [28] It shows the native ESI mass spectra of trimeric AtIspF incubated with 4-diphosphocytidyl-2C-methyl-D-erythritol 2-phosphate (CDP-MEP; L) (Trimer : L ratio $=1: 0$ to 1 : 2 ) in the absence and presence of $\mathrm{Zn}^{2+}$ ( $a, b$ and e, f, respectively). Catalytic activity of the trimeric enzyme in the presence of $\mathrm{Zn}$ cofactor releases cytidine monophosphate (CMP). A zoom into the most intense signal (Trimer ${ }^{14+}$ ) is shown next to the individual spectra. A native ESI mass spectrum of the bare protein is given for reference (a). AtIspF was incubated with CDP-MEP (Trimer : L ratio of $1: 2$ ) in the absence (c, d) and presence of $\mathrm{Zn}^{2+}(\mathrm{g}, \mathrm{h})$, red and black markers indicate the number of CMP or CDP-MEP molecules bound to AtIspF, respectively. Fig. $4 \mathrm{c}$ and $\mathrm{d}$ show that the incubation of zinc-deficient AtIspF with the substrate yields complexes containing up to three CDP-MEP molecules bound to the protein trimer. On the other hand, saturating AtIspF with $\mathrm{Zn}^{2+}$ results in a mass shift corresponding to three $\mathrm{Zn}^{2+}$ ions bound to the protein (Fig. 4e and f). Upon incubation of $\mathrm{Zn}^{2+}$-saturated AtIspF with CDP-MEP, fine-structured complex peaks were observed with a mass shift of approx. 322 Da that match with the mass of CMP, one of the products of the reaction
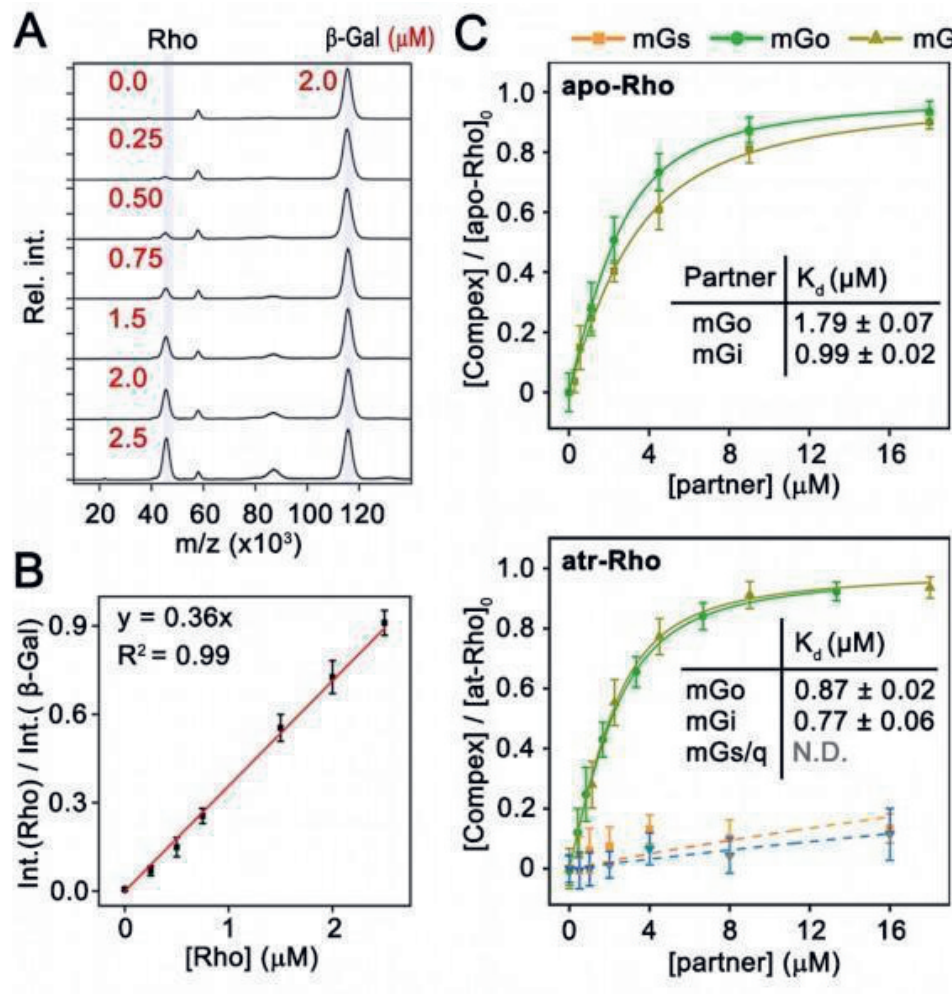

Fig. 3. Determination of binding affinities between GPCRs and partner proteins. (A) Calibration of different concentrations of Rho normalized to $2 \mu \mathrm{M}$ of $\beta$-galactosidase. (B) Peak intensity ratio of Rho to $\beta$-galactosidase vs. Rho concentration in the sample. (C) Evaluation of the affinities (dissociation constants $\mathrm{K}_{\mathrm{d}}$, measured in $\mu \mathrm{M}$ ) for apo-Rho and Rho bound to the agonist alltrans retial with mGo (green) and mGi (beige). Rho does not bind to $\mathrm{mGs}$ and $\mathrm{mGa}$ (blue \& yellow). (D) Crystal structure of the Rho•mGo complex (PDB: 2I37) Reproduced with permission from ref. [27]. Copyright 2021, National Academy of Science.

\section{Protein Complexes studied by Native ESI-MS}

MALDI-MS in linear/high-mass mode is often used when working with large protein complexes, because it produces spectra dominated by singly charged ions that are simple to interpret, and unlike ESI is not prone to suppression effects by low-mass additives such as salts, buffers, or detergents. However, as evident from Figs 2 and 3 it has a fairly poor mass resolution, making it hard to detect the binding between small partners (MW $\left.<3^{\prime} 000\right)$ and proteins. We have therefore also studied proteinligand interactions using ESI-MS. Fig. 4 presents an example,
(Fig. 4g and h). Ions corresponding to free CDP-MEP (c) or CMP (g), protonated or with sodium cations bound, were found in the mass spectrum. The signal intensity in spectra $\mathrm{c}$ and $\mathrm{g}$ is magnified 10-fold in the range from 300 to $600 \mathrm{~m} / z$. The data in this figure underscores that significantly higher mass resolution is necessary to unravel all the different chemical ligation states that play a role in this catalytic process, especially for quite complex oligomeric proteins such as IspF with multiple ligand binding sites. 


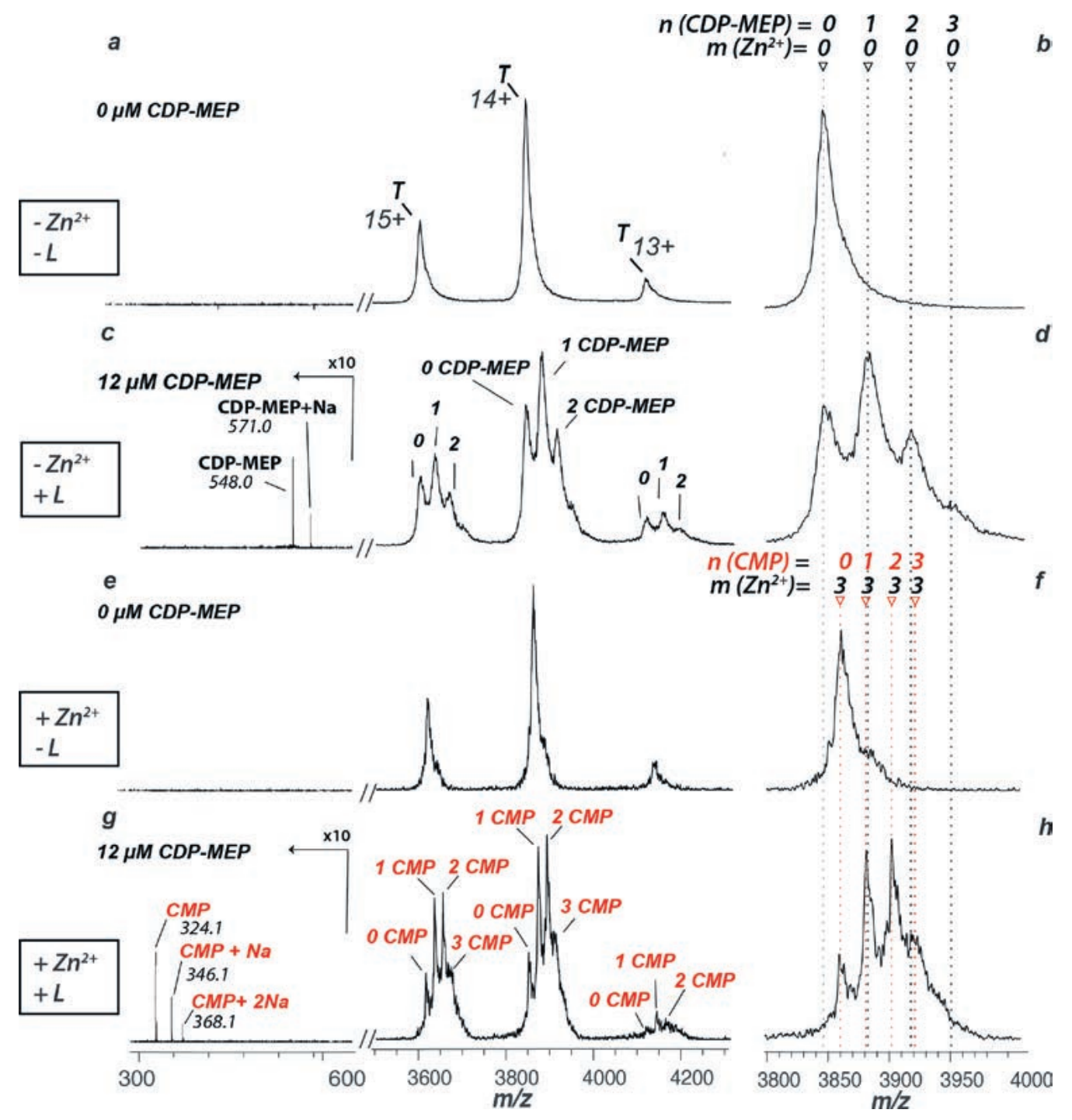

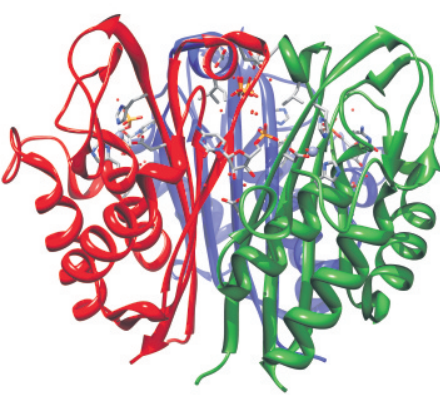

Fig. 4. Native ESI mass spectra of AtlspF in the presence and absence of ligand and zinc. For details, see text. A ribbon diagram (side view) representing the X-ray structure of AtlspF in complex with CMP and $\mathrm{Zn}^{2+}$ shown as stick models is given above. (PDB entry 2PMP). Reproduced with permission from ref. [28].

\subsection{Native ESI-MS for Measuring $K_{D}$}

Our group has also been active in studying noncovalent complexes quantitatively by native ESI-MS, using titration experiments, to obtain noncovalent binding constants. This work has recently been extended to a high-throughput format involving the 'gap sampler' (Fig. 5). ${ }^{[29]}$ It was used to rapidly mix and electrospray small molecule ligands together with a target protein and compare the screening results with data from thermal shift assay (TSA) and surface plasmon resonance (SPR). In a first round, all three techniques were used for a screening of 110 ligands against bovine carbonic anhydrase II (CA II), which resulted in five mutual hits and some false positives with ESI-MS, presumably due to the high ligand concentration and interferences from dimethyl sulfoxide (DMSO). In a second round, 33 compounds were screened in lower concentration and in a less complex matrix, resulting in only true positives with ESI-MS. Within a cycle time of $30 \mathrm{~s}$, dissociation constants were determined within an order of magnitude accuracy consuming only 5 pmol of ligand and less than 15 pmol of protein per screened compound. In a third round, dissociation constants of five compounds were accurately determined in a titration experiment. Quantitation was possible by using an internal standard (IS) for normalization (Fig. 5). Thus, the gap sampler can rapidly and efficiently be used for high-throughput screening as well as for $\mathrm{K}_{\mathrm{d}}$ determination.

\subsection{Investigation of Capsid Forming Proteins and Protein Complexes}

Another activity of our lab has been the study of the capsid forming proteins such as lumazine synthase from Aquifex aeolicus (AaLS-13), ${ }^{[30]}$ the iron-storing protein ferritin ${ }^{[31]}$ and bacterial microcompartments (BMC) like $\mathrm{CO}_{2}$-fixing carboxysome, which is composed of many different proteins, ${ }^{[32,33]}$ using modern MS methods. In an investigation of the carboxysome's constituents, formation of various heterohexamers of $\mathrm{CcmKi}$ isomers along with multiple protein complexes, e.g. $\mathrm{CcmO} \cdot \mathrm{CcmK} 2$ were investigated using native ESI-MS. As shown in Fig. 6a, the carboxysome is an icosahedron protein shell made of various proteins that assemble themselves into pentameric and hexameric pores allowing specifically $\mathrm{CO}_{2}$ and $\mathrm{HCO}_{3}{ }^{-}$molecules to be upconcentrated for the Calvin cycle. In addition to monomer analysis of each $\mathrm{CcmKi}$ isoforms, the mass spectra of heterogeneous hexamers of $\mathrm{CcmK} 1 / \mathrm{K} 2$ and CcmK3/K4 were recorded and shown in Fig. 6c and d, right side. These spectra illustrate that not only icosahedral planes can not only be heterogeneous and made from different hexamers and pentamers, but hexamers themselves can be diversified and form a mosaic. This structural complexity is challenging and leads to complications in deconvolution analysis since the peaks in the high $\mathrm{m} / \mathrm{z}$ region are broad and unresolved (Fig. 6c, d). This is further illustrated by the spectrum on the left showing our attempt to analyze $\mathrm{CcmO} \cdot \mathrm{CcmK} 2$ protein complexes (Fig. 6b, right side). Although the identification of several charge states in 


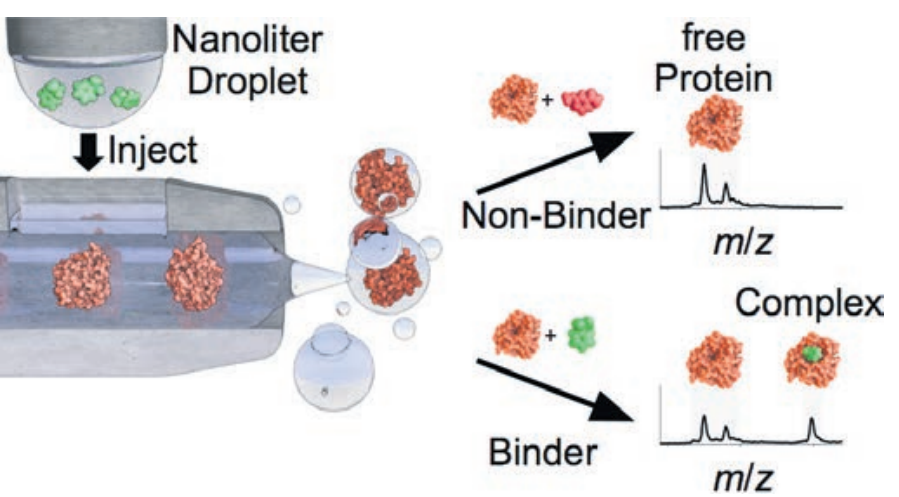

Fig. 5. (top) Principle of the gap-sample based high-throughput binding assays. (right) Example of one injection cycle for ligand $L$ that binds to the protein P, bovine carbonic anhydrase. (a) shows the extracted ion current (XIC) during one injection cycle of a high affinity ligand for the IS, the complex signal $[\mathrm{P}+\mathrm{L}]^{+10}$ and the free protein $\mathrm{P}^{+10}$. All $\mathrm{XIC}$ drop to zero during the injection because the spray becomes momentarily unstable. After a few seconds of residence time, the IS and $[\mathrm{P}+\mathrm{L}]^{+10}$ signals raise while the $\mathrm{P}^{+10}$ signal from the unbound protein is decreasing. The dashed line marks the point when the mass spectrum is read out for $\mathrm{K}_{\mathrm{d}}$ calculation, i.e., where the bound to unbound ratio $\mathrm{R}$ is maximal. (b) shows exactly this mass spectrum. At low $\mathrm{m} / \mathrm{z}$ the IS is observed and at high $\mathrm{m} / \mathrm{z}$ different charge states of both bound and unbound $\mathrm{P}$ are visible. (c) shows a zoom into the region of the +10 charge state. $L$ and acetate (Ac) complexes are observed. Reproduced with permission from ref. [29], J. Kaeslin et al., Anal. Chem. 2021, 93, 13342. Copyright 2021 American Chemical Society.
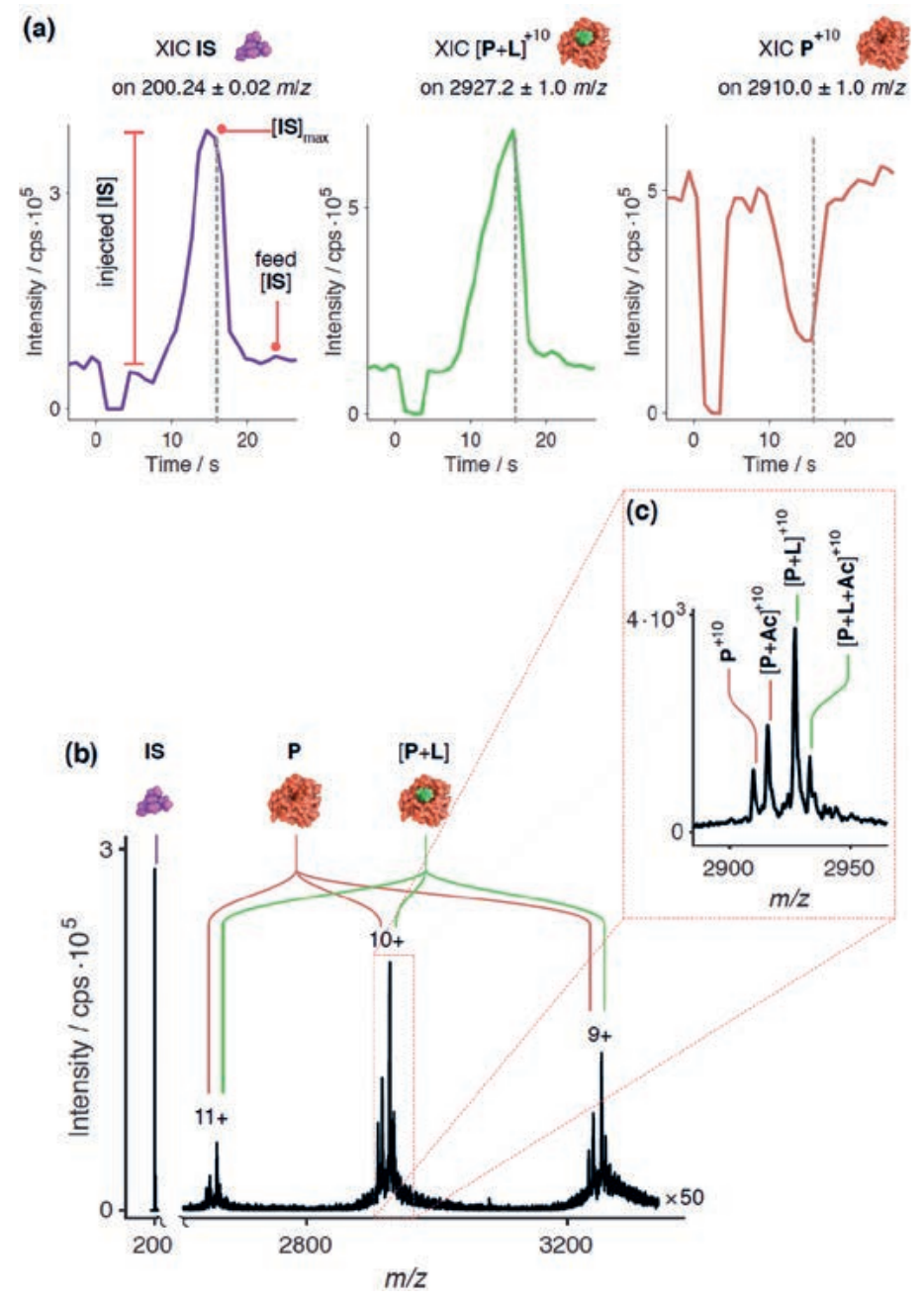

\subsection{Studying Non-canonical Nucleic Acid Structures with T-programmed ESI-MS}

Another example for the use of novel technology concerns investigation of the melting and disassembly behavior of DNA G-quadruplexes. These are non-canonical nucleic acid structures essential for cellular processes such as DNA replication, transcription, translation, and telomere shortening. Hybrid quadruplexduplex oligonucleotide assemblies composed of multiple domains are challenging to study with conventional biophysical methods due to their structural complexity. We carried out thermal denaturation experiments to investigate the unfolding of multi-stranded oligonucleotide constructs derived from biologically relevant structures, and to identify unfolding intermediates, in a single experiment (Fig. 7). ${ }^{[34]}$ The melting temperatures $T_{m}$ and the thermodynamic characteristics of proximal DNA domains were found to be influenced by the number of proximal domains, their position, and their order. Fig. $7 \mathrm{a}$ and $\mathrm{b}$ compare the information obtained by circular dichroism (CD) spectroscopy of such a system with MS data. It is obvious that much more detail can be obtained from the latter

For example, the stability of a three-domain system, the complex of a G-Quadruplex-duplex forming single strandG-quadruplex construct (G-D-G) in the presence of a complementary strand and $1 \mathrm{mM} \mathrm{KCl}$ was investigated (Fig. 7c). At room temperature, the duplex domain between two folded GQs was clearly present, but unfolded subsequently, as shown in Fig. $7 \mathrm{~d}$. The thermal denaturation via temperature-controlled ESI-MS showed the presence of an intermediate at $\sim 58^{\circ} \mathrm{C}$. Based on the mass difference, we associated this intermediate to an assembly containing just one fully folded GQ. Remarkably, Van’t Hoff analysis of this data revealed that the formation of the central spectra were too broad and unresolved in the higher $m / z$, range to draw any conclusion. 

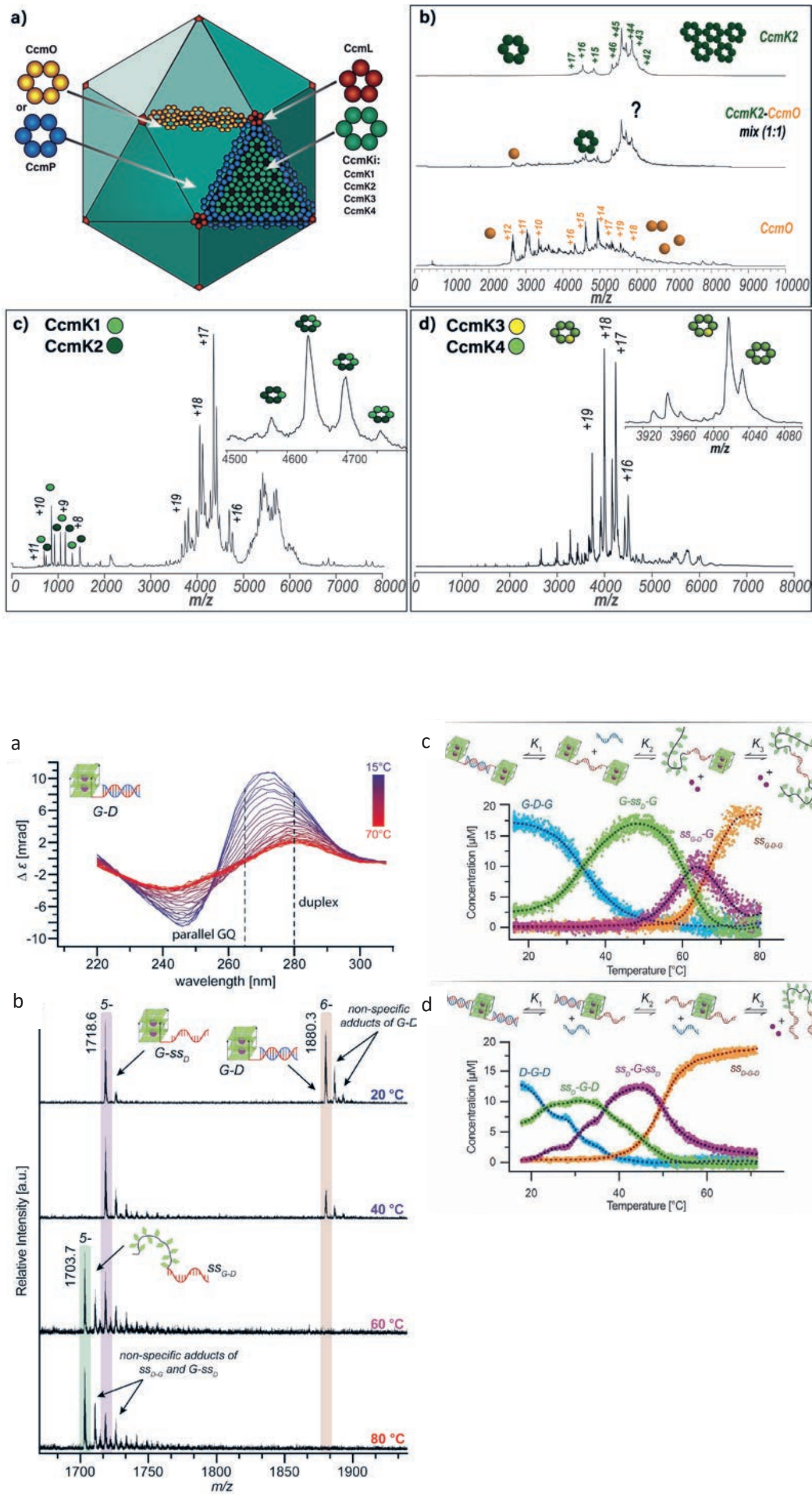

Fig. 6. Summary of the progress in our research on carboxysome capsids, indicating the complexity of this heterogeneous protein micro-compartment, and displaying key mass spectral data. a) Schematic of the carboxysome, showing an icosahedral structure made from various isoforms of CcmK proteins forming hexamers, pentamers of $\mathrm{CcmL}$, and $\mathrm{CcmO} / \mathrm{CcmL}$ pseudo hexamers on the edges. b) Native mass spectrum demonstrates the attempt to study high mass sections of carboxysome like CcmO-CcmK2 proteins complex. c) and d) Two mass spectra point out that hexamers themselves can be mosaic and diversified and hard to resolve. Reproduced with permission from ref. [33] and adapted from ref. [34].
Fig. 7 (a) Comparison of $C D$ and mass spectra of single-domain and two-domain DNA complexes at different temperatures. (b) CD spectra of two-domain G-D complex showing spectral overlap of the individual domain CD profiles. Dashed lines highlight wavelengths chosen for $\mathrm{CD}$ melting (duplex $-280 \mathrm{~nm}$, fully parallel $\mathrm{GQ}-265 \mathrm{~nm}$ ). (b) Mass spectra of G-D complex at different temperatures. As labelled, the observed signals correspond to the folded complex $(\mathrm{m} / \mathrm{z}=1880.3)$, a semi-folded intermediate $(\mathrm{m} / \mathrm{z}$ $=1718.6)$, and the completely unfolded complex $(\mathrm{m} / \mathrm{z}=1703.7)$. (c; d) Thermal denaturation profiles acquired using TCnESI-MS. Samples of $20 \mu \mathrm{M}$ (C) G-D-G and (D) D-G-D mixed with $20 \mu \mathrm{M}$ complementary strand and $1 \mathrm{mM}$ $\mathrm{KCl}$. Melting curves represent fully folded complex (॰), first intermediate $(\bullet)$, second intermediate (๑), and fully unfolded complex (॰). Images for Fig. 7 a-d taken from ref. [35]. Adapted from ref. [35]. Reproduced with permission from Wiley-VCH. 
duplex restabilized both GQs and increased their $\mathrm{T}_{\mathrm{m}}$ by $5-7^{\circ} \mathrm{C}$, although the Gibbs free energy diagram of the G-D-G complex showed only small energetic differences between single domains and domains in the G-D-G complex. ${ }^{[35]}$

\subsection{ESI-MS Investigation of Post-translational Modification of Proteins and their Noncovalent Binding Profiles}

The glycoform distributions on specific protein residues are another interest of our lab, as they play a crucial role in affecting the affinity of protein॰protein or protein $\bullet$ cell interactions. Alterations of these glycan patterns, naturally occurring during disease or in biomanufacturing processes, are still difficult to quantify and require highly reproducible and non-destructive methods. In recent years, our group has developed several quantitative, mass spectrometric approaches to determine the carbohydrate distribution of specific proteins. Isotope labeling with succinic anhydride was demonstrated to be an efficient tool in quantifying a specific protein glycosylation profile using MALDI-MS and LC-ESI-MS. ${ }^{[36]}$ Following this study, our group further developed a label-free approach for rapid $\mathrm{N}$-glycosylation determination on monoclonal antibodies. For this, and for monitoring $\mathrm{N}$-glycan profiles from monoclonal IgGs, produced by a running perfusion bioreactor, ${ }^{[37]}$ a microarray-based MS method that was originally developed in our laboratory for singlecell MALDI and metabolomics studies.

Another interesting example is the Fc $\gamma \mathrm{RIIIa} / \mathrm{CD} 16 \mathrm{a}$, a receptor used to enhance the antibody-dependent cell cytotoxicity (ADCC) mechanism via glycoengineered monoclonal antibodies (mAbs). Its extracellular domain has five potential $N$-glycosylation sites, ${ }^{[38]}$ with two directly involved in $\mathrm{mAb} F \mathrm{~F}$ fragment recognition. In a very recent study, we showed an interaction analysis of glycoengineered antibodies with the CD16a receptor, using native mass spectrometry. ${ }^{[39]}$ Fig. 8 presents a native ESI spectrum of CD16a analyzed using ESI-MS. As the glycosylation patterns play a crucial role in protein protein interaction, the complex formation of native CD16a and different glycosylation states of its wild-type monoclonal antibody were investigated. Recently, our laboratory deployed a new high-resolution ESI-IMS-MS instrument that is currently being used to disentangle the different carbohydrate patterns on this receptor protein, which were previously non-resolvable.

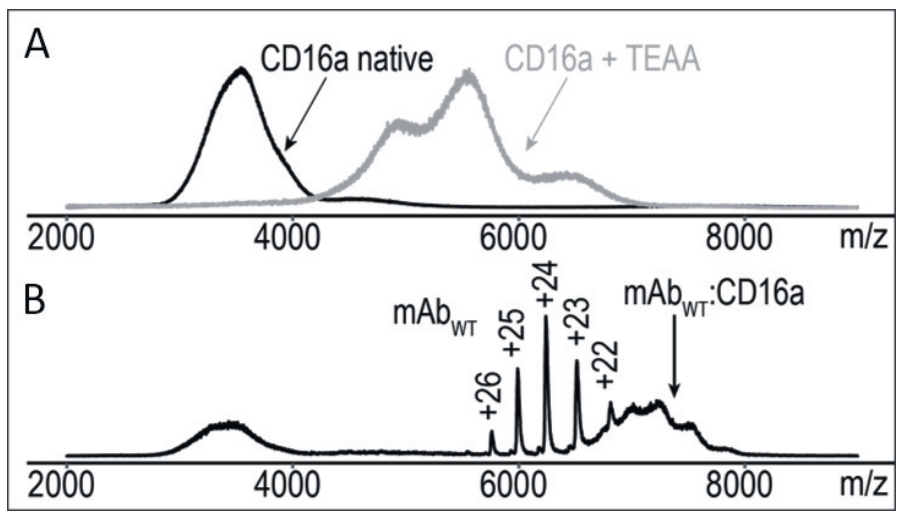

Fig. 8 a) Native ESI-MS spectrum of CD16a in native conditions without (left) and with triethylammonium acetate (TEAA, right), recorded on our hybrid quadrupole TOF mass spectrometer (applied $\mathrm{m} / \mathrm{z}$ mass range: 1000-15000). Due to its five $\mathrm{N}$-glycosylation sites, overlapping signals from heterogenous CD16a prevented a correct charge state identification. b) In complex with its wild type glycosylated monoclonal, a CD16a•mAbWT complex can be monitored at higher $\mathrm{m} / \mathrm{z}$ ratio. Reproduced with permission from ref. [39]; copyright 2020, Taylor \& Francis.

\section{Conclusions and Outlook}

While soft ionization mass spectrometry is now firmly established, the study of noncovalent complexes by MS is still in a phase of rapid development. In part, this is fueled by technological developments like the ones discussed in this paper, but also others, for example, Orbitrap instruments with high-mass capabilities, or high-resolution ion mobility devices that are interfaced to highresolution mass spectrometers. Increasingly, these technologies allow very difficult structural problems to be studied in unprecedented detail. An interesting challenge that I believe remains for the mass spectrometry community is to educate fellow scientists working in supramolecular chemistry or structural biology about the capabilities of these novel methods. They can all become very useful to solve problems that are difficult to address with conventional biophysical methods.

\section{Acknowledgments}

The author would like to thank many talented postdocs, $\mathrm{PhD}$ students and MSc students who have worked on the above projects, and the Swiss National Science Foundation (current grant \# 200020_178765) for continuous and very generous support of this research over many years.

Received: November 5, 2021

[1] J. R. Bolla, M. T. Agasid, S. Mehmood, C. V. Robinson, Annu. Rev. Biochem. 2019, 88, 85, https://doi.org/10.1146/annurev-biochem-013118-111508.

[2] V. Gabelica, A. Shvartsburg, C. Afonso, P. Barran, J. Benesch, C. Bleiholder, M. Bowers, A. Bilbao, M. Bush, J. Campbell, I. Campuzano, T. Causon, B. Clowers, C. Creaser, E. De Pauw, J. Far, F. Fernandez-Lima, J. Fjeldsted, K. Giles, M. Groessl, C. Hogan Jr, S. Hann, H. Kim, R. Kurulugama, J. May, J. McLean, K. Pagel, K. Richardson, M Ridgeway, F. Rosu, F. Sobott, K. Thalassino, S. Valentine, T. Wyttenbach, Mass Spectrom. Rev. 2019, 38, 291, https://doi.org/10.1002/mas.21585.

[3] J. Y. Han, T. S. Choi, C. E. Heo, M. K. Son, H. I. Kim, Mass. Spec. Rev 2019, 38, 483, https://doi.org/10.1002/mas.21596.

[4] W. B. Struwe, C. V. Robinson, Curr. Opin. Struc. Biol. 2019, 58, 241, https://doi.org/10.1016/j.sbi.2019.05.019.

[5] M. Zhou, C. Lanz, K. A. Brown, Y. Ge, L. Paša-Tolić, J.A. Loo, F. Lermyte, Chem. Sci. 2020, 11, 12918, https://doi.org/10.1039/d0sc04392c.

[6] J. L. Bennett, G. T. H. Nguyen, W. A. Donald, Chem. Rev. 2021; in press, https://doi.org/10.1021/acs.chemrev.1c00293.

[7] J. E. Keener, G. Z. Zhang, M. T. Marty, Anal. Chem. 2021, 93, 583, https://doi.org/10.1021/acs.analchem.0c04342.

[8] T. Y. Low, S. E. Syafruddin, M. A. Mohtar, A. Vellaichamy, N. S. A Rahman, Y.-F. Pung, C. S. H. Tan, Cell. Mol. Life Sci. 2021, 78, 5325, https://doi.org/10.1007/s00018-021-03856-0.

[9] A. L. Richards, M. Eckhardt, N. J. Krogan, Mol. Syst. Biol. 2021, 17, e8792, https://doi.org/10.15252/msb.20188792.

[10] N. Tamara, M. A. de Boer, A. J. R. Heck, Chem. Rev. 2021; in press, https://doi.org/10.1021/acs.chemrev.1c00212.

[11] S. Ghiasikhou, M. Fabricio da Silva, Y. Zhu, R. Zenobi, Anal. Bioanal. Chem. 2017, 409, 6873, https://doi.org/10.1007/s00216-017-0652-8.

[12] S. Ghiasikhou, A. H. Marchand, R. Zenobi, Microfluid. Nanofluid. 2019, 23 60, https://doi.org/10.1007/s10404-019-2229-7.

[13] S. Ghiasikhou, S. Cazzamalli, J. Scheuermann, D. Neri, R. Zenobi, Anal. Bioanal. Chem. 2019, 411, 7387, https://doi.org/10.1007/s00216-019-02165-7.

[14] A. Marchand, M. Czar, E. Eggel, J. Kaeslin, R. Zenobi, Nature Commun. 2020, 566, 1, https://doi.org/10.1038/s41467-019-14179-x.

[15] N. Wu, L. Jiao, M. Bütikofer, Z. Zeng, R. Zenobi, Anal. Chem. 2021, 93, 10982, https://doi.org/10.1021/acs.analchem.1c02126.

[16] V. Neu, R. Steiner, S. Müller, C. Fattinger, R. Zenobi, Anal. Chem. 2013, 85 , 4628, https://doi.org/10.1021/ac400186t.

[17] V. Neu, P. Dörig, C. Fattinger, S. Müller, R. Zenobi, Microfluid. Nanofluid. 2016, 20, 62, https://doi.org/10.1007/s10404-016-1732-3.

[18] J. L. P. Benesch, F. Sobott, C. V. Robinson, Anal. Chem. 2003, 75, 2208 , https://doi.org/10.1021/ac034132x.

[19] G. Wang, R. R. Abzalimov, I. A. Kaltashov, Anal. Chem. 2011, 83, 2870 , https://doi.org/10.1021/ac200441a.

[20] J. W. McCabe, M. Shirzadeh, T. E. Walker, C.-W. Lin, B. J. Jones, V. H. Wysocki, D. P. Barondeau, D. E. Clemmer, A. Laganowsky, D. H. Russell, Anal. Chem. 2021, 93, 6924, https://doi.org/10.1021/acs.analchem.1c00870.

[21] K. J. Laszlo, J. H. Buckner, E. B. Munger, M. F. Bush, J. Am. Soc. Mass Spectrom. 2017, 28, 1382, https://doi.org/10.1007/s13361-017-1620-4. 
[22] T. J. El-Baba, D. W. Woodall, S. A. Raab, D. R. Fuller, A. Laganowsky, D. H. Russell, D. E. Clemmer, J. Am. Chem. Soc. 2017, 139, 6306, https://doi.org/10.1021/jacs.7b02774.

[23] K. Jeanne Dit Fouque, F. Fernandez-Lima, J. Phys. Chem. B 2020, 124, 6257, https://doi.org/10.1021/acs.jpcb.0c04276.

[24] B. Hommersom, T. Porta, R. M. A. Heeren, Int. J. Mass Spectrom. 2017, 419, 52, https://doi.org/10.1016/j.ijms.2017.03.008.

[25] M. Köhler, C. Neff, C. Perez, C. Brunner, E. Pardon, J. Steyaert, G. Schneider, K. Locher, R. Zenobi, Anal. Chem. 2018, 90, 5306, https://doi.org/10.1021/acs.analchem.8b00236.

[26] N. Wu, L. Jiao, M. Bütikofer, Z. Zeng, R. Zenobi, Anal. Chem. 2021, 93, 10982, https://doi.org/10.1021/acs.analchem.1c02126.

[27] N. Wu, A.M. Olechwier, C. Brunner, P. Edwards, C.-J. Tsai, C. Tate, G.F.X. Schertler, G. Schneider, X. Deupi, R. Zenobi, P.-Y. Ma, Proc. Nat. Acad. Sci. USA 2021, 118, e2024146118, https://doi.org./10.1073/pnas.2024146118.

[28] K. Root, K. Barylyuk, A. Schwab, J. Thelemann, B. Illarionov, J. Geist, T. Gräwert, A. Bacher, M. Fischer, F. Diederich, R. Zenobi, Chem. Sci. 2018, 9 , 5976, https://doi.org/10.1039/C8SC00814K.

[29] J. Kaeslin, C. Brunner, S. Ghiasikhou, G. Schneider, R. Zenobi, Anal. Chem. 2021, 93, 13342, https://doi.org/10.1021/acs.analchem.1c03130.

[30] K. Root, R. Frey, D. Hilvert, R. Zenobi, Helv. Chim. Acta 2017, 100, e1700166, https://doi.org/10.1002/hlca.201700166.

[31] J. Lan, R. Zenobi, Rapid Commun. Mass Spectrom. 2019, 33, 1855, https://doi.org/10.1002/rcm.8546

[32] L. F. Garcia-Alles, E. Lesniewska, K. Root, N. Aubry, N Pocholle, C. I. Mendoza, E. Bourillot, K. Barylyuk, D. Pompon, R. Zenobi, D. Reguera, G. Truan, Plos One 2017, 12, e018109, https://doi.org/10.1371/journal.pone.0185109.

[33] L. F. Garcia-Alles, K. Root, L. Maveyraud, N. Aubry, E. Lesniewska, L. Mourey, R. Zenobi, G. Truan, Plos One 2019, 14, e0223877, https://doi.org/10.1371/journal.pone.0223877.
[34] K. Root, 'Dissecting Non-covalent Interactions in Oligomeric Protein Complexes Using Native Mass Spectrometry', Ph.D. thesis \# 25170, ETH Zurich, 2018, https://doi.org/10.3929/ethz-b-000283762.

[35] A. Pruška, A. Marchand, R. Zenobi, Angew. Chem. Int. Ed. 2021, 60, 15390, https://doi.org/10.1002/anie.202016757.

[36] M. Pabst, I. Benesova, S. Fagerer, M. Jacobsen, K. Eyer, G. Schmidt, R. Steinhoff, J. Krismer, F. Wahl, J. Preisler, R. Zenobi, J. Proteome Res. 2016, 15, 326, https://doi.org/10.1021/acs.jproteome.5b00899.

[37] J. Hajduk, M. Wolf, R. Steinhoff, D. Karst, J. Souquet, H. Broly, M. Morbidelli, R. Zenobi, J. Biotechnol. 2019, 302, 77, https://doi.org/10.1016/j.jbiotec.2019.06.306.

[38] P. G. Subedi, A. W. Barb, J. Biol. Chem. 2018, 293, 16842, https://doi.org/10.1074/jbc.RA118.004998.

[39] J. Hajduk, C. Brunner, S. Malik, J. Bangerter, G. Schneider, M. Thomann, D. Reusch, R. Zenobi, mAbs 2020, 12, 1736975 , https://doi.org/10.1080/19420862.2020.1736975.

\section{License and Terms}

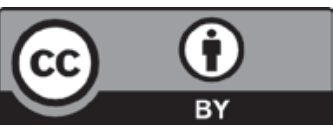

This is an Open Access article under the terms of the Creative Commons Attribution License CC BY 4.0. The material may not be used for commercial purposes.

The license is subject to the CHIMIA terms and conditions: (https://chimia.ch/chimia/about).

The definitive version of this article is the electronic one that can be found at https://doi.org/10.2533/chimia.2022.101 